International Journal of Modern Physics C

Vol. 26, No. 10 (2015) 1550109 (17 pages)

(c) The Authors

DOI: $10.1142 /$ S0129183115501090

\title{
A hybrid artificial bee colony algorithm for numerical function optimization
}

\author{
Zakaria N. Alqattan* and Rosni Abdullah ${ }^{\dagger}$ \\ School of Computer Sciences \\ Universiti Sains Malaysia \\ Penang, 11800, Malaysia \\ *zqttan2@yahoo.com \\ †rosni@cs.usm.my
}

Received 18 November 2014

Accepted 9 January 2015

Published 27 February 2015

\begin{abstract}
Artificial Bee Colony (ABC) algorithm is one of the swarm intelligence algorithms; it has been introduced by Karaboga in 2005. It is a meta-heuristic optimization search algorithm inspired from the intelligent foraging behavior of the honey bees in nature. Its unique search process made it as one of the most competitive algorithm with some other search algorithms in the area of optimization, such as Genetic algorithm (GA) and Particle Swarm Optimization (PSO). However, the $\mathrm{ABC}$ performance of the local search process and the bee movement or the solution improvement equation still has some weaknesses. The ABC is good in avoiding trapping at the local optimum but it spends its time searching around unpromising random selected solutions. Inspired by the PSO, we propose a Hybrid Particle-movement ABC algorithm called HPABC, which adapts the particle movement process to improve the exploration of the original ABC algorithm. Numerical benchmark functions were used in order to experimentally test the HPABC algorithm. The results illustrate that the HPABC algorithm can outperform the ABC algorithm in most of the experiments ( $75 \%$ better in accuracy and over 3 times faster).
\end{abstract}

Keywords: Swarm intelligence algorithms; artificial bee colony algorithm; particle swarm optimization; numerical function optimization.

PACS Nos.: 11.25.Hf, 123.1K.

\section{Introduction}

Nowadays, the swarm intelligence based optimization algorithms are among the most interesting and effective algorithms. These algorithms are inspired by observing the behavior of the fish schools, birds' flocks and insect colonies like ant, termites and bee colonies when they seek for food. The swarm intelligence is defined by Bonabeau et al. (1999) as "... .any attempt to design algorithms or distributed problem solving devices inspired by the collective behavior of social insects colonies and other animal societies...". ${ }^{1}$ The swarm intelligence based algorithms had shown a good 
performance in the last few years. Thus, most of the recent works are focused on using these algorithms on different types of real-world optimization problems. ${ }^{2}$ In the recent years, many swarm intelligence algorithms have been proposed, such as Particle Swarm Optimization (PSO), ${ }^{3}$ Ant Colony Optimization (ACO), ${ }^{4}$ Bacterial Foraging Optimization (BFO),,$^{5}$ Artificial Fish Swarm Algorithm (AFSA) ${ }^{6}$ and Artificial Bee Colony (ABC) algorithm. ${ }^{7}$ Bees Colony Optimization algorithm is one of the problem solving algorithms that have been applied on search optimization problems. The Bees Colony has many features which can be performed in order to model the intelligent search systems such as "bee dance (communication), bee foraging, queen bee, task selection, collective decision making, nest site selection, mating, floral/pheromone laying, navigation systems". 8

ABC algorithm has been invented by Karaboga in 2005, inspired from the foraging behavior of the bee in nature. ${ }^{7}$ It has been used widely in the recent years on different kinds of optimization problems. First, it was used on numerical benchmark problems, ${ }^{1,9}$ on constrained optimization problems in Ref. 10, for training neural networks, ${ }^{11}$ Bahamish 2009 used the ABC for protein 3D structure prediction, ${ }^{12}$ on fuzzy clustering to classify different data sets, ${ }^{13}$ also to solve the TSP problem, ${ }^{14}$ and as a feature selection for biomarker Discovery in mass spectrometry analysis. ${ }^{15}$ Moreover, authors in Ref. 16, also used the ABC algorithm for protein 3D structure prediction with main chain angles based method, their results showed that the ABC algorithm has an efficient performance on improving the protein 3D prediction.

However, the ABC algorithm is like any known heuristic "due to the randomization involved in their respective initial steps" algorithm, it has advantages and also some disadvantages. The ABC has the capability to increase the probability of finding the optimal solution and avoid falling into the local optimal by performing the local and global search in every generation and has fewer control parameters compared to other algorithms. ${ }^{17,18}$ On the other hand, there are insufficiencies in ABC algorithm, due to its simple operation based on solution improvement process, the convergence speed of the algorithm is increased. ${ }^{18}$ Therefore, the ABC algorithm causes a fast convergence and can be trapped in the local optimum for some complex multimodal problems. ${ }^{19}$ On the contrary, the algorithm's information exchange process is being performed on one dimension with random neighbor in each solution improvement; thus, the convergence speed decreases as the dimension of the problem increases. ${ }^{20}$ In addition, the information sharing activity of the ABC models showed a weak performance during the experiments. ${ }^{21}$ However, it is impossible to find an optimization algorithm that can reach the global optimum for every optimization problem. $^{22}$

In order to address the disadvantages, several variants of $\mathrm{ABC}$ have been proposed to enhance the exploration and exploitation capability, convergence speed and avoid being trapped at the local optimum. ${ }^{16-18,20,23-29}$ The modifications include changes on the ABC itself or hybridizing it with other optimization algorithms. Reference 29 was among the recent works involving the modification of the ABC algorithm, where a selection mechanism is added after the employed bee phase so 
that only a number of solutions will be stored in a separate memory for the next phase. Then the Onlooker bees will select solutions from the separated memory to be improved. Most of the modifications are focused on the ABC's solution improvement or information sharing process which is the main factor for the algorithm's exploration and exploitation capability. In this paper, we propose to improve this factor by applying the PSO algorithm's solution improvement or information sharing process.

Particle swarm optimization (PSO) is one of the competitive swarm intelligence algorithms in the area of the heuristic search and optimization problems. It has been applied on many real-time problems. ${ }^{30-44}$ Its mechanism of solution improvement and information sharing procedure are unique and differ from that used in the ABC algorithm. It consists of using special parameter called Velocity $(v)$, which is used to control the degree of improvement to be conduct on the solution based on its previous state. With two other parameters called $c_{1}$ and $c_{2}$, which are learning factors that determine the relative influence of cognitive (self-confidence) and social (swarm-confidence) components, respectively (Eq. (1)).

$$
V_{i, d}^{t+1}=w^{t} * V_{i, d}^{t}+c_{1}^{t} * r_{1} *\left(\text { best }_{i, d}^{t}-X_{i, d}^{t}\right)+c_{2}^{t} * r_{2} *\left(\text { best }_{i, d}^{t}-X_{i, d}^{t}\right),
$$

where $w$ represents inertia weight, $c_{1}$ and $c_{2}$ are learning factors which determine the relative influence of cognitive (self-confidence) and social (swarm-confidence) components, respectively, $r_{1}$ and $r_{2}$ are independent random numbers uniformly distributed in the range $[0,1] . V_{i, d}^{t+1}, X_{i, d}^{t}$ and $p$ best $_{i, d}^{t}$ are the velocity, position and the personal best of $i$ th particle in $d$ th dimension for the $t$ th iteration, respectively. The $g$ best $_{i, d}$ is the $d$ th dimension of best particle in the swarm for the $t$ th iteration. These parameters are the key subject of the information sharing strategy that the PSO perform during the search process. The PSO information sharing strategy is very different from the ABC strategy (Eq. (2)), and it provides more information about the search space including the local best solution information sharing and the global best as well. In ABC algorithm the new position of the chosen solution is calculated by the following equation:

$$
x_{i(j+1)}=x_{i j}+\beta\left(x_{i j}-x_{k j}\right),
$$

where $i$ represents the particular solution (food source) position $(i=1,2, \ldots, N), k$ represents the neighbor's randomly chosen solution position $(k=1,2, \ldots, N)$, the $k$ value should be different from the $i$ value. $\beta$ is a random number between $[-1,1]$ used to estimate the neighbor food sources around $x_{i j}$. After a number of ABC specified search cycles the food source with unimproved fitness will be abandoned, and the scout bee will be sent to find a new food source based on Eq. (3). Then the food source found by the scout bee will take the place of the abandoned one. ${ }^{1}$

$$
x_{i j}=x_{i j}+\operatorname{rand}[0,1]\left(x_{j \max }-x_{j \min }\right) .
$$

In this paper, a novel Hybrid Particle-move $\mathrm{ABC}$ algorithm is proposed to improve and enhance the exploration capability of the classical ABC algorithm. The work is 
based on using the particle moving strategy with the ABC algorithm which will lead the search process to more efficient and substantial results. The rest of the paper is organized as follows: Section 2 presents the proposed HPABC algorithm. Section 3 illustrates the types of the experiments conducted and the implementation results of the proposed algorithm. The conclusion and future work are discussed in Sec. 4.

\section{The Proposed HPABC}

Basically our inspiration comes from the particles movement operation in the Particle Swarm Optimization (PSO) algorithm. In PSO the particles moves or do their local search based on three main categories first: The particles next move rely on the Velocity variable which means it is not a random or an arbitrary movement, second: The local information based strategy which comes from the Local Best solution variable which interacts with the chosen particle's next move value and third: The Global Best solution variable which also has a great effect on the particle's next move; while the Artificial Bee Colony (ABC) algorithm has none of them. The bee local search or solution improvement is based totally on a magnitude determined randomly from a random chosen neighbor solution, as in Eq. (2). This means that the added value on the trial solution in each loop is totally random (while the PSO has the Velocity variable). The ABC's local search process has no information based procedure; the best solution discovered so far is not always held in the population since it might be replaced with a randomly produced solution by a scout. Therefore, it might not contribute to the production of trial solutions. ABC local search focuses only on which solution will be selected for the improvement part (local search) at the "employed bees" stage and at "onlooker bees" stage as well, but the solutions with the higher fitness value are used more often than those with less fitness values to produce trial solutions in "onlooker bees" stage. In other words, the Onlooker bees Phase has one difference that it relies on the probability value of the solution in order to select it. On the other hand, the global search mechanism done by the scout provides the $\mathrm{ABC}$ algorithm with the ability to prevent the search from premature convergence problem. This ability is not present in the PSO algorithm search process. However, in PSO the best solution found so far is always kept in the population and it can be used for producing new solutions (with new velocities). It means that the promising regions of the search space are searched in shorter time and in detail. But this may cause the algorithm to be trapped in the local minima; that is why we kept the limit parameter of the ABC in the proposed HPABC. The limit parameter prevents the original $\mathrm{ABC}$ from being trapped in the local minima by inserting a random selected solution into the search space from time to time. In the proposed algorithm $\mathrm{HPABC}$ we complement the advantages of the $\mathrm{ABC}$ algorithm with the advantages of the PSO algorithm in the optimization search problems. However, some researchers consider the ABC as more efficient than the PSO in term of the accuracy while the PSO is faster than the ABC in term of the execution time, for some optimization problems. ${ }^{45}$ 
In the HPABC algorithm, the colony of the artificial bee contains only two groups of bees: onlookers and scouts. The hall colony members are of onlooker bees. In other words, the number of the food sources is equal to the number of the colony size. The onlooker bee of an abandoned food source becomes a scout. Note that the employed bees phase is eliminated in HPABC because basically it is similar to the onlooker bees' phase with more randomization strategy where there is no probability determination for the selected solution. Moreover, it is almost doubling the search time consumption of the original ABC algorithm. The search carried out by the HPABC artificial bees is illustrated as follows:

- Onlooker bees determine food sources in the search space and share their information with each other within the hive and then select one of the food sources.

- The selected food source then being investigated for new food source using Eq. (1).

- An onlooker bee of which the source has been abandoned becomes a scout and starts to search for new food source randomly using Eq. (3).

The HPABC algorithm main steps are as follows:

Initialization step: The same process as the original ABC and PSO algorithms. REPEAT

Move the Onlookers onto the food sources and determine their nectar amounts. Move the scouts to search for new food sources replacing the abandoned ones. Memorize the best food source found so far.

UNTIL (requirements are met).

Each cycle of the HPABC search consist of three mean steps: calculate the probability of the food sources which are currently in the search space then randomly select the food source with the higher fitness (based on the probability) to be searched by the onlooker bees (note: the food source with higher fitness maybe selected more than ones). At the onlooker phase Eq. (1) will be used instead of Eq. (2) for the local search. Then send the scout bee to search for new food sources for those how are exceed the limit parameter (similar to the original ABC), using Eq. (3).

\section{Experiments}

In this section, we will present the experimental steps, the strategies and the results in order to complete the evaluation process of the proposed HPABC algorithm. This section contains the numerical benchmark functions description, the parameters settings experiments of HPABC, the original ABC and PSO parameters settings and the HPABC evaluation results.

\subsection{Benchmark functions}

In order to investigate the performance of the $\mathrm{HPABC}$ algorithm compared to $\mathrm{ABC}$ and PSO algorithms on numerical function optimization problems, six numerical 
benchmark functions used in Refs. 9-11 are used here. Which can be categorized into multimodal (with separable and nonseparable variables) and unimodal functions (with separable and nonseparable variables).

The first function is the Griewank function described by

$$
f_{1}(\mathbf{x})=1 / 4000\left(\sum_{i=1}^{D}\left(x_{i}-100\right)^{2}-\left(\Pi_{i=1}^{D} \cos \left(x_{i}-100 / \sqrt{i}\right)\right)\right)+1,
$$

where $\mathbf{x}=\left[x_{1}, x_{2}, \ldots, x_{D}\right]$, the initial range of $\mathbf{x}$ is $[-600,600]^{D}$ and $D$ denotes the diminution of the solution space. The minimum solution of the Griewank function is $\mathbf{x}^{*}=[100,100, \ldots, 100]$ and $f_{1}\left(\mathbf{x}^{*}\right)=0$. It is a multimodal function with non-separable variables.

The second function is the Sphere function described by

$$
f_{2}(\mathbf{x})=\sum_{i=1}^{D} x_{i}^{2}
$$

where the initialization range of $\mathbf{x}$ is $[-100,100]^{D}$. The minimum solution of the Sphere function is $\mathbf{x}^{*}=[0,0, \ldots, 0]$, and $f_{2}\left(\mathbf{x}^{*}\right)=0$. It is a unimodal function with separable variables.

The third function is the Rosenbrock function described by

$$
f_{3}(\mathbf{x})=\sum_{i=1}^{D-1} 100\left(x_{(i+1)}-x_{i}^{2}\right)^{2}+\left(x_{i}-1\right)^{2}
$$

where the initialization range of $\mathbf{x}$ is $[-15,15]^{D}$. The minimum solution of the Rosenbrock function is $\mathbf{x}^{*}=[1,1, \ldots, 1]$, and $f_{3}\left(\mathbf{x}^{*}\right)=0$. It is a unimodal function with nonseparable variables. Its global optimum is inside a long, narrow, parabolic shaped flat valley. So it is difficult to converge to the global optimum.

The fourth function is the Rastrigin function described by

$$
f_{4}(\mathbf{x})=\sum_{i=1}^{D}\left(x_{i}^{2}-10 \cos \left(2 \pi x_{i}\right)+10\right),
$$

where the initialization range of $\mathbf{x}$ is $[-15,15]^{D}$. The minimum solution of the Rastrigin function is $\mathbf{x}^{*}=[0,0, \ldots, 0]$ and $f_{4}\left(\mathbf{x}^{*}\right)=0$. It is a multimodal function with separable variables.

The fifth function is the Ackley function described by

$$
f_{5}(\mathbf{x})=20+e-20 \exp \left(-0.2 \sqrt{1 / D \sum_{i=1}^{D} x_{i}^{2}}\right)-\exp \left(1 / D \sum_{i=1}^{D} \cos \left(2 \pi x_{i}\right)\right)
$$

where the initialization range of $\mathbf{x}$ is $[-32.768,32.768]^{D}$. The minimum solution of the Ackley function is $\mathbf{x}^{*}=[0,0, \ldots, 0]$ and $f_{5}\left(\mathbf{x}^{*}\right)=0$. It is a multimodal function with nonseparable variables. 
The sixth function is the Schwefel function described by

$$
f_{6}(\mathbf{x})=\sum_{i=1}^{D}-x_{i} \sin \left(\sqrt{\left|x_{i}\right|}\right),
$$

where the initialization range of $\mathbf{x}$ is $[-500,500]^{D}$. The minimum solution of the Schwefel function is $\mathbf{x}^{*}=[420.96,420.96, \ldots, 420.96]$ and $f_{6}\left(420.96^{*}\right)=418.9829 D$. It is a multimodal function with separable variables.

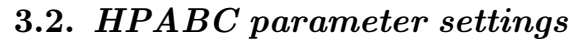

In order to set the parameters for the new proposed HPABC algorithm we need to do some experimental tests on the algorithm to investigate the parameters settings issue before we move on to the numerical function optimization test. As a new proposed algorithm the parameter should go under test experiments to illustrate the best parameters settings for the algorithm. The main parameters that have the great influence in this algorithm are: $w, c_{1}, c_{2}$ and limit where $w$ represents inertia weight, $c_{1}$ and $c_{2}$ are learning factors which determine the relative influence of cognitive (local best) and social (global best) components, limit is diversity control parameter of the algorithm, respectively. Therefore, we experimentally try to determine those parameter values by changing the value of a parameter and fix the values of the others. Also, we select one of the numerical function problems in order to perform the test on it. Griewank function is the function we choose for the test, the $D$ value was set to 30 , HPABC population number was 80, iteration number was 5000 (the number of FEs is 400000 ) and each of the experiments was repeated 30 times independently for each parameter value exchange. The experiments was implemented on a normal PC with ADM Athlon(tm) 7750 Dual-Core processor 2.70 GHz CPU and 4.00 GB RAM. Table 1 illustrates the scenarios we propose for the parameter $w$ test.

From Table 1, we can conclude that the best average result $(2.22 \mathrm{E}-16)$ of Griewank function is achieved when the $w$ parameter equals to 0.3 and when $\left(c_{1}, c_{2}=\right.$ 1.5 and $\left.c_{1}, c_{2}=2\right)$. The second best average result was $(2.37 \mathrm{E}-016)$ when $w$ equals

Table 1. HPABC parameter $w$ test scenarios $($ limit $=100)$.

\begin{tabular}{|c|c|c|c|c|c|c|}
\hline \multirow[b]{2}{*}{$w$} & \multicolumn{2}{|c|}{$c_{1}, c_{2}=1$} & \multicolumn{2}{|c|}{$c_{1}, c_{2}=1.5$} & \multicolumn{2}{|c|}{$c_{1}, c_{2}=2$} \\
\hline & Mean & Std.dev. & Mean & Std.dev. & Mean & Std.dev. \\
\hline 0.1 & $2.92 \mathrm{E}-16$ & $6.07 \mathrm{E}-17$ & $2.59 \mathrm{E}-16$ & $5.23 \mathrm{E}-17$ & $2.55 \mathrm{E}-16$ & $5.84 \mathrm{E}-17$ \\
\hline 0.2 & $2.74 \mathrm{E}-16$ & $6.24 \mathrm{E}-17$ & $2.26 \mathrm{E}-16$ & $3.49 \mathrm{E}-17$ & $2.37 \mathrm{E}-16$ & $3.77 \mathrm{E}-17$ \\
\hline 0.3 & $2.41 \mathrm{E}-16$ & $4.14 \mathrm{E}-17$ & $2.22 \mathrm{E}-16$ & $2.87 \mathrm{E}-17$ & $2.22 \mathrm{E}-16$ & $4.05 \mathrm{E}-17$ \\
\hline 0.4 & $2.37 \mathrm{E}-16$ & $3.77 \mathrm{E}-17$ & $2.33 \mathrm{E}-16$ & $5.98 \mathrm{E}-17$ & $2.48 \mathrm{E}-16$ & $6.20 \mathrm{E}-17$ \\
\hline 0.5 & $2.41 \mathrm{E}-16$ & $4.14 \mathrm{E}-17$ & $2.59 \mathrm{E}-16$ & $6.62 \mathrm{E}-17$ & $3.44 \mathrm{E}-16$ & $9.66 \mathrm{E}-17$ \\
\hline 0.6 & $2.89 \mathrm{E}-16$ & $8.41 \mathrm{E}-17$ & $3.77 \mathrm{E}-16$ & $9.33 \mathrm{E}-17$ & $4.66 \mathrm{E}-16$ & $9.24 \mathrm{E}-17$ \\
\hline 0.7 & $4.44 \mathrm{E}-16$ & $8.11 \mathrm{E}-17$ & $8.25 \mathrm{E}-16$ & $8.55 \mathrm{E}-16$ & $3.80 \mathrm{E}-07$ & $1.01 \mathrm{E}-06$ \\
\hline 0.8 & $5.27 \mathrm{E}-14$ & $1.47 \mathrm{E}-13$ & 0.274055 & 0.339218 & 0.456532 & 0.410207 \\
\hline 0.9 & 2.3923 & 0.910195 & 3.31303 & 1.4914 & 1.53146 & 0.847508 \\
\hline
\end{tabular}


Table 2. Parameter $w=0.3$ and $w=0.4$ test scenarios (limit $=100)$.

\begin{tabular}{lccccc}
\hline & \multicolumn{2}{c}{$w=0.3, c_{2}=1.5$} & & \multicolumn{2}{c}{$w=0.4, c_{2}=1.5$} \\
\cline { 2 - 3 } \cline { 5 - 6 }$c_{1}$ & Mean & Std.dev. & & Mean & Std.dev. \\
\hline 1.1 & $2.33 \mathrm{E}-16$ & $3.33 \mathrm{E}-17$ & & $2.41 \mathrm{E}-16$ & $4.14 \mathrm{E}-17$ \\
1.2 & $2.33 \mathrm{E}-16$ & $4.39 \mathrm{E}-17$ & & $2.33 \mathrm{E}-16$ & $5.25 \mathrm{E}-17$ \\
1.3 & $2.37 \mathrm{E}-16$ & $5.54 \mathrm{E}-17$ & & $2.33 \mathrm{E}-16$ & $4.39 \mathrm{E}-17$ \\
1.4 & $2.29 \mathrm{E}-16$ & $3.99 \mathrm{E}-17$ & & $2.37 \mathrm{E}-16$ & $5.54 \mathrm{E}-17$ \\
1.5 & $2.29 \mathrm{E}-16$ & $2.77 \mathrm{E}-17$ & & $2.18 \mathrm{E}-16$ & $5.35 \mathrm{E}-17$ \\
1.6 & $2.33 \mathrm{E}-16$ & $3.33 \mathrm{E}-17$ & & $2.26 \mathrm{E}-16$ & $3.49 \mathrm{E}-17$ \\
1.7 & $2.33 \mathrm{E}-16$ & $5.25 \mathrm{E}-17$ & $2.55 \mathrm{E}-16$ & $5.09 \mathrm{E}-17$ \\
1.8 & $2.29 \mathrm{E}-16$ & $4.91 \mathrm{E}-17$ & & $2.29 \mathrm{E}-16$ & $4.91 \mathrm{E}-17$ \\
1.9 & $2.18 \mathrm{E}-16$ & $5.35 \mathrm{E}-17$ & & $2.41 \mathrm{E}-16$ & $5.03 \mathrm{E}-17$ \\
$\mathbf{2}$ & $\mathbf{2 . 1 5 E}-\mathbf{1 6}$ & $\mathbf{3 . 9 9 E}-\mathbf{1 7}$ & $2.74 \mathrm{E}-16$ & $7.44 \mathrm{E}-17$ \\
\hline
\end{tabular}

to 0.4 and $c_{1}, c_{2}=1$. It can also be recognized that the HPABC is a stable algorithm when we look at the Standard Deviation (Std.dev.) values, which is very close to zero in most of the results achieved during the tests.

In order to decide which value is more efficient with $w$ parameter to get better results, we suggested more investigation on the proposed HPABC algorithm's performance; the local best and global best parameters $\left(c_{1}, c_{2}\right)$ are assigned for experiments. We changed the values of the parameters $c_{1}, c_{2}$ to find the best results, as: First we will start with the parameter $c_{1}$ to test its effects on the $w$ parameter. (Note: because it is a heuristic search we attempted to select the best average result that has a smallest Std.dev.), as the scenarios in Table 2. The best result of Griewank function which can be seen in Table 2 is $(2.15 \mathrm{E}-016)$ when $w=0.3$ and $c_{1}=2, c_{2}=1.5$, and we can see from the Std.dev. values, the algorithm is stable and most of the global minimum results are close to each other. Now we will perform the same test experiments with the parameter $c_{2}$ but when the parameter $c_{1}=2$ as resulted from Table 2. From Table 3, the best result of Griewank function is

Table 3. Parameters $w=0.3$ and $w=0.4, c_{1}=2$ test scenarios (limit $=100)$.

\begin{tabular}{lccccc}
\hline & \multicolumn{2}{c}{$w=0.3, c_{1}=2$} & & \multicolumn{2}{c}{$w=0.4, c_{1}=2$} \\
\cline { 2 - 3 } \cline { 5 - 6 }$c_{2}$ & Mean & Std.dev. & & Mean & Std.dev. \\
\hline 1.1 & $2.33 \mathrm{E}-16$ & $4.39 \mathrm{E}-17$ & & $2.37 \mathrm{E}-16$ & $5.54 \mathrm{E}-17$ \\
1.2 & $2.22 \mathrm{E}-16$ & $2.87 \mathrm{E}-17$ & & $2.37 \mathrm{E}-16$ & $6.24 \mathrm{E}-17$ \\
1.3 & $2.26 \mathrm{E}-16$ & $1.99 \mathrm{E}-17$ & & $2.52 \mathrm{E}-16$ & $6.37 \mathrm{E}-17$ \\
1.4 & $2.33 \mathrm{E}-16$ & $4.39 \mathrm{E}-17$ & & $2.52 \mathrm{E}-16$ & $4.91 \mathrm{E}-17$ \\
$\mathbf{1 . 5}$ & $\mathbf{2 . 1 1 E}-\mathbf{1 6}$ & $\mathbf{4 . 3 9 E}-\mathbf{1 7}$ & & $2.59 \mathrm{E}-16$ & $7.76 \mathrm{E}-17$ \\
1.6 & $2.33 \mathrm{E}-16$ & $4.39 \mathrm{E}-17$ & & $2.52 \mathrm{E}-16$ & $6.37 \mathrm{E}-17$ \\
1.7 & $2.26 \mathrm{E}-16$ & $3.49 \mathrm{E}-17$ & & $2.26 \mathrm{E}-16$ & $6.07 \mathrm{E}-17$ \\
1.8 & $2.33 \mathrm{E}-16$ & $3.33 \mathrm{E}-17$ & & $2.59 \mathrm{E}-16$ & $7.21 \mathrm{E}-17$ \\
1.9 & $2.41 \mathrm{E}-16$ & $5.79 \mathrm{E}-17$ & & $2.33 \mathrm{E}-16$ & $5.98 \mathrm{E}-17$ \\
2 & $2.29 \mathrm{E}-16$ & $4.91 \mathrm{E}-17$ & $2.44 \mathrm{E}-16$ & $6.66 \mathrm{E}-17$ \\
\hline
\end{tabular}


$(2.11 \mathrm{E}-016)$ when $w=0.3$ and $c_{1}=2, c_{2}=1.5$, which is the best results from all the previous experimental tests. Finally we can conclude that these are the best parameters settings for the proposed HPABC algorithm which enable it to achieve the best average results for the Griewank function. Moreover, it can be illustrated that the less impact of the global best parameter $\left(c_{2}\right)$ on the algorithm's search performance is better.

In other words, the more the search relies on the information provided by the local best is improving the search rather than considering the information from the global best parameter. It is also important to declare that this results explicitly indicated that the improvement on the individuals (solutions) in the search space of the algorithm during the local search is rapidly increase due to parameter $c_{1}$ (learning factor which determines the relative influence of cognitive "local best"); which can be seen from the results in Tables 2 and 3 when the parameter $c_{1}$ is set on high values. However, when the parameter $c_{2}$ (learning factor which determines the relative influence of social "global best") increased to large values it will negatively affects the individuals' (solutions) improvement in the search space. In other words the improvement of the local search space during the onlooker bees phase is fast enough to almost dispense the need for the global best information provided in Eq. (4). In addition, the Std.dev. (4.39E-017) when $w=0.3, c_{1}=2$ and $c_{2}=1.5$ is more close to zero. This means that the results are more stable when $w=0.3$ than when $w=0.4$

For the final parameters testing of the HPABC, we experimentally test the parameter limit by changing its value to indicate if it will affect the search performance of the proposed algorithm. Table 4 shows the results of parameter limit tests. From Table 4, the limit parameter test has shown there is no big difference between the results after the various changes on limit value when $D=30$, but when $D=60$ there is a clear improvement in the result while the limit is increasing (see Table 4). This may be due to the type of the function we used (Griewank function). Because it is a heuristic search algorithm, the search may differ from one problem to another and also from one algorithm run settings to another. Therefore, we assume that the best average value of limit for this algorithm is (300). In conclusion, from Tables $1-4$, we can identify that the best parameters settings for the HPABC algorithm are

Table 4. Change the limit parameter with $w=0.3$, $c_{1}=2$ and $c_{2}=1.5$.

\begin{tabular}{lcclll}
\hline & \multicolumn{2}{c}{$D=30$} & & \multicolumn{2}{c}{$D=60$} \\
\cline { 2 - 3 } \cline { 5 - 6 } limit & Mean & Std.dev. & & Mean & Std.dev. \\
\hline 100 & $2.11 \mathrm{E}-16$ & $4.39 \mathrm{E}-17$ & & $8.92 \mathrm{E}-16$ & $1.09 \mathrm{E}-16$ \\
200 & $2.18 \mathrm{E}-16$ & $3.49 \mathrm{E}-17$ & & $6.74 \mathrm{E}-16$ & $1.03 \mathrm{E}-16$ \\
300 & $2.00 \mathrm{E}-16$ & $5.29 \mathrm{E}-17$ & & $6.74 \mathrm{E}-16$ & $1.07 \mathrm{E}-16$ \\
400 & $2.18 \mathrm{E}-16$ & $3.49 \mathrm{E}-17$ & & $6.25 \mathrm{E}-16$ & $8.35 \mathrm{E}-17$ \\
500 & $2.07 \mathrm{E}-16$ & $3.77 \mathrm{E}-17$ & $6.37 \mathrm{E}-16$ & $9.48 \mathrm{E}-17$ \\
\hline
\end{tabular}


$(w=0.3, c 1=2, c 2=1.5$, limit $=300)$ for this experiment. These settings will be used for evaluation of HPABC algorithm in the following section. In addition, it is important to mention that this type of the optimization search is a meta-heuristic search and the proposed HPABC is based on a meta-heuristic optimization search algorithms. This indicates that the results illustrated by the previous experiments may not be exact values always, and this is why in such type of optimization search the researchers prefer using the Mean and the Std.dev. for results presentation.

\section{3. $A B C$ \& $P S O$ parameters settings}

Many comparative experiments were done on some of the multi-dimensional numerical optimization function in order to improve the function minimum results and also test the new invented algorithms or even modified algorithms. ${ }^{9,17,19-21,23,24,26,28,29,46-48}$ In this section, a set of experiments tested on the six numerical benchmark functions were performed to compare the performance of the HPABC with that of other algorithms. In order to analytically investigate the new proposed HPABC algorithm, two algorithms the original ABC and the original PSO algorithms will be used for the comparison. Moreover, the main parameters of all the algorithms such as: population size, maximum number of generation and the number of runs for each experiments are set to $(80,5000,30)$ respectively, as in Ref. 46. While the PSO parameters were as follows: Learning factors $c 1=c 2=2$, velocity lower and upper bounds $V_{\min }=0.1 * X_{\min }, V_{\max }=0.1 * X_{\max }{ }^{20}$ and $w=0.4$.

However, to fairly compare the performance of the original ABC with the proposed HPABC, we will set the parameter limit $=300$ also in the original ABC algorithm. The parameter limit has a great influence on the algorithm's population diversity, which helps the algorithm to avoid premature convergence and to investigate different regions during the search. On the other hand, the limit also has a great influence on the algorithms results; a high limit value gives the solution extra opportunity to be selected for more improvement in the next iteration, which causes the algorithm to investigate more on each solution in the search space and find a better neighbor solutions.

\subsection{HPABC evaluation results}

In this section, the evaluation of the HPABC will be illustrated through the numerical benchmark functions results. Table 4 shows the optimization results of the Griewank function, Sphere function, Rosenbrock function, Rastrigin function, Ackley function and Schaffer function, respectively. The experiments follows the settings used by Ref. 46, two kinds of dimensions of solution space were tested for each function. All the functions were tested with dimension 30 and 60 . The population size was set on 80 and maximum number of generation was 5000 for all algorithms. That means the number of the function evaluations is 400000 . All experiments were repeated for 30 times independently for each function with different random seeds. The mean function values of the best solutions found by the 
algorithms for different dimensions have been recorded. The Mean, standard deviations Std.dev., Best, Worst, Time_mean and Time_Std.dev are the optimization results of the six test functions which are shown in Table 5, and the best results obtained for each function is written in bold. ${ }^{\mathrm{a}}$

The results of HPABC are better or very close to the best results of the two other algorithms (ABC and PSO). All the detailed results are shown in Table 5. And for example, we performed a single run of all the algorithms on all the selected functions (see Fig. 1). By only considering the Function Evaluation number regardless of the consumed time of each algorithm and when $D=30$, the results were as follows: Griewank and Ackley functions are two multimodal nonseparable variables functions, on Griewank function PSO algorithm showed the best performance and reached the global optima at $80000 \mathrm{FEs}$. While the HPABC has slower convergence than PSO and reached the global optimum at $160000 \mathrm{FEs}$. The ABC was the worst and has slow convergence attitude and trapped on the local minimum at $120000 \mathrm{FEs}$, as shown in Fig. 1(a). The search performance order was PSO $>$ HPABC $>$ ABC. On Ackley function the results of the algorithms were very close to each other, the search performance order was PSO $>\mathrm{HPABC}>\mathrm{ABC}$ as shown in Fig. 1(b). Sphere and Rosenbrock are two unimodal with nonseparable variables functions. On Sphere function all the algorithms obtained satisfactory results because it is a simple problem. However, the PSO algorithm showed the best performance as shown in Fig. 1(c). The search performance order was PSO $>$ HPABC $>$ ABC. On Rosenbrock function, the $\mathrm{ABC}$ algorithm showed the best performance and has the faster convergence to the global minimum, where the HPABC has the next better result which is very close to the ABC result. The worst result was obtained by the PSO algorithm. It has slow convergence speed and trapped on the local minimum from the earlier stages, the search performance order was $\mathrm{ABC}>\mathrm{HPABC}>\mathrm{PSO}$, as shown in Fig. 1(d). Rastrigin and Schwefel functions are two multimodal with separable variables functions, on Rastrigin function the HPABC algorithm achieved the best result and has the faster convergence speed to the global minimum value, it converged fast after $80000 \mathrm{FEs}$ and reached the global optima (0). The ABC algorithm had the next best result and reached the global optima at $120000 \mathrm{FEs}$. While the PSO algorithm showed the worst result and trapped fast in the local minimum at the earlier stages of the search (20000 FEs). The search performance order was HPABC $>$ ABC $>$ PSO, as shown in Fig. 1(e). The Schwefel function, the ABC and the HPABC algorithms had almost the same performance result, but the HPABC showed a little improvement in the earlier stages of the search where it converges faster than the ABC, as shown in Fig. 1(f). While the PSO algorithm showed the worst result with slow convergence and trapped at the local minimum value. The search performance order was $\mathrm{HPABC}>\mathrm{ABC}>\mathrm{PSO}$.

Table 5 shows that the results obtained from the proposed HPABC algorithm are equal or close to the optimal ones and the standard deviations are relatively small. It

a The best results between $\mathrm{ABC}$ and $\mathrm{HPABC}$ are highlighted in bold. 


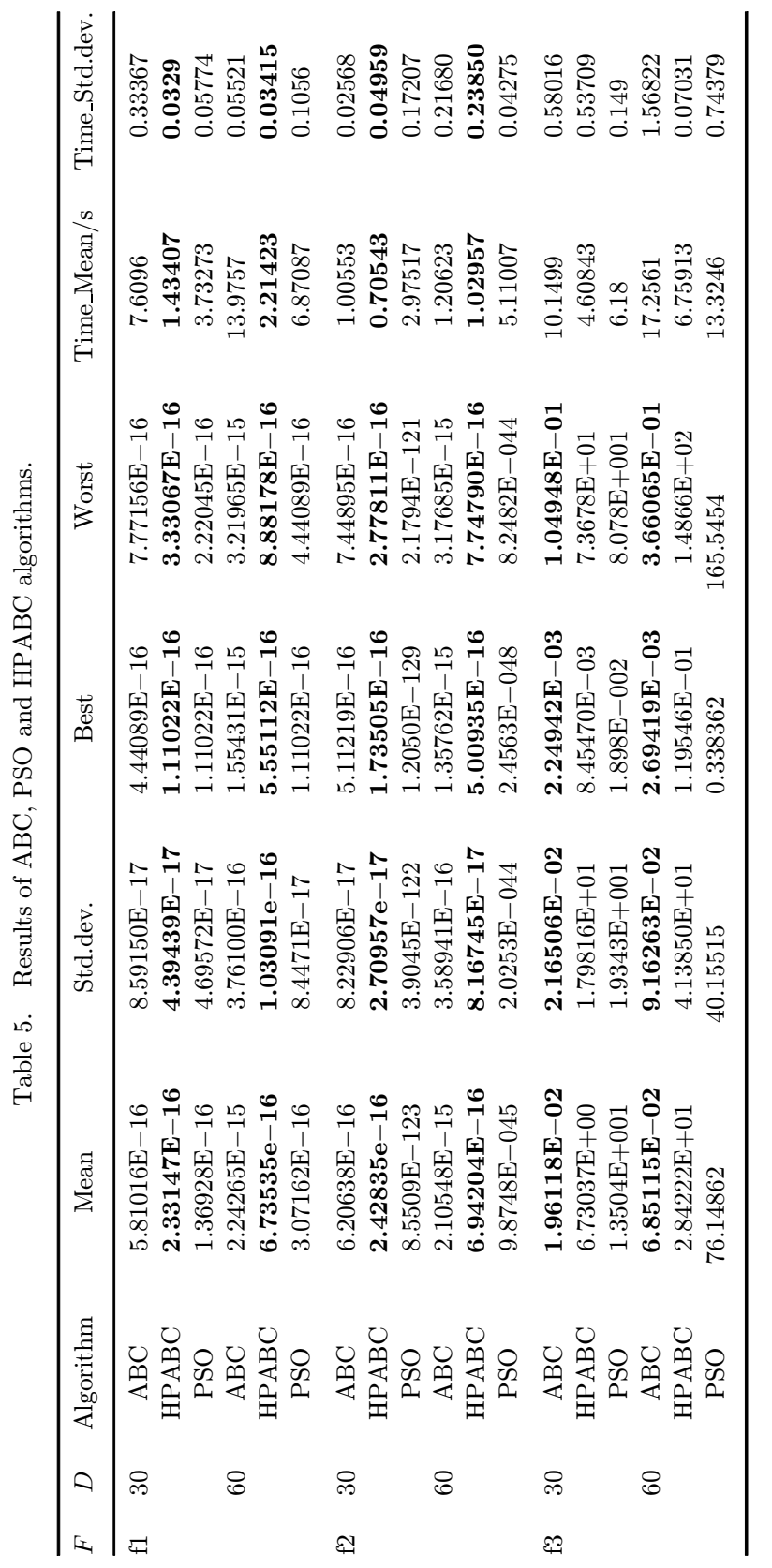




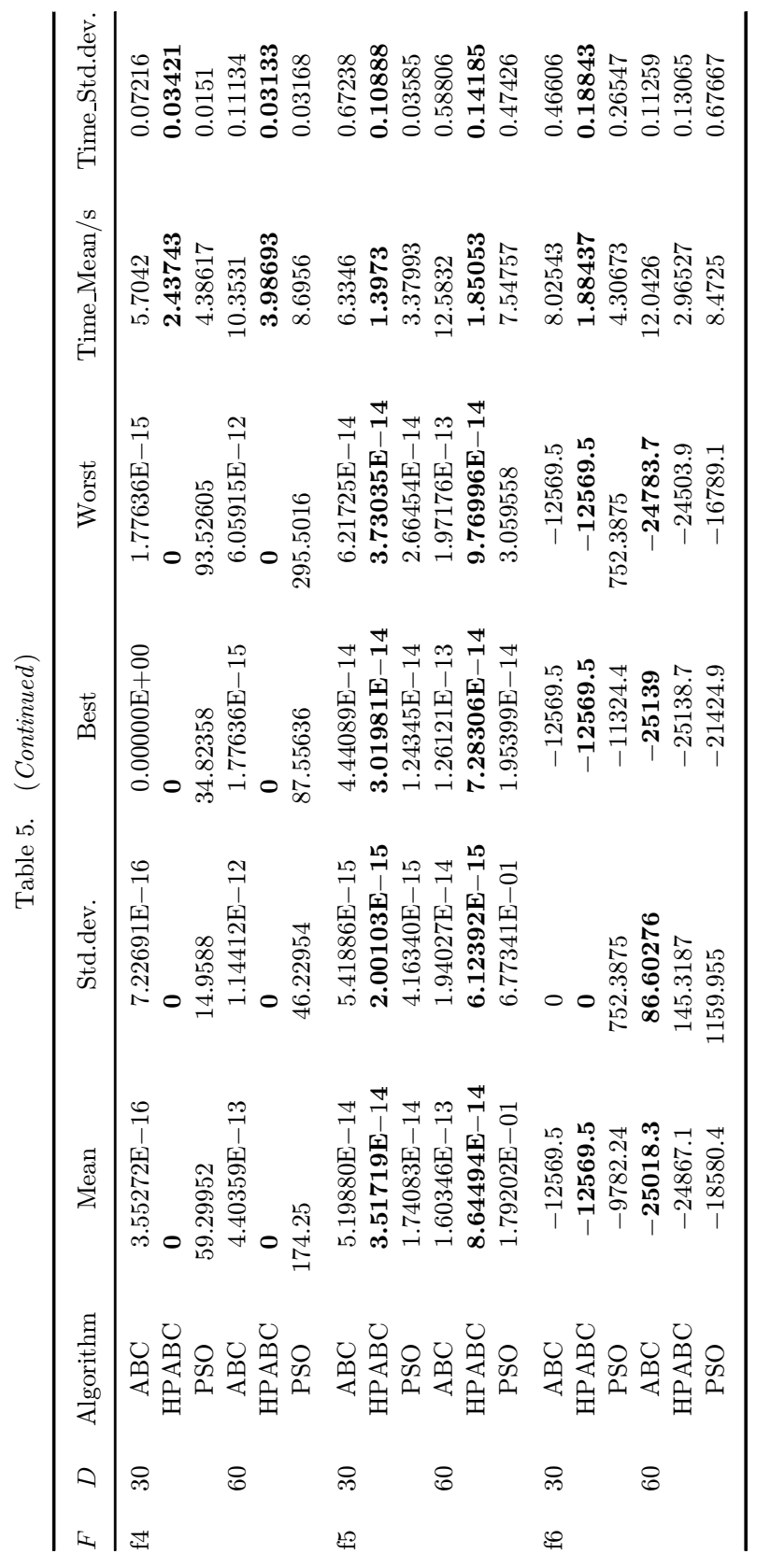




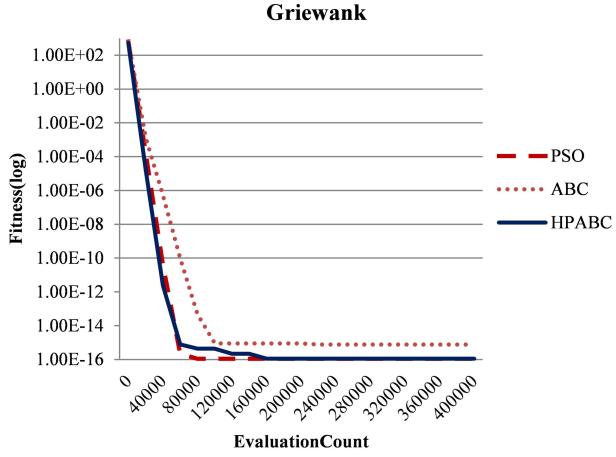

(a)

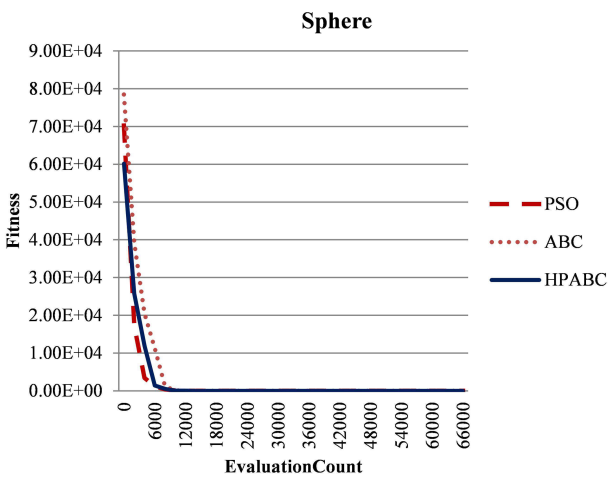

(c)

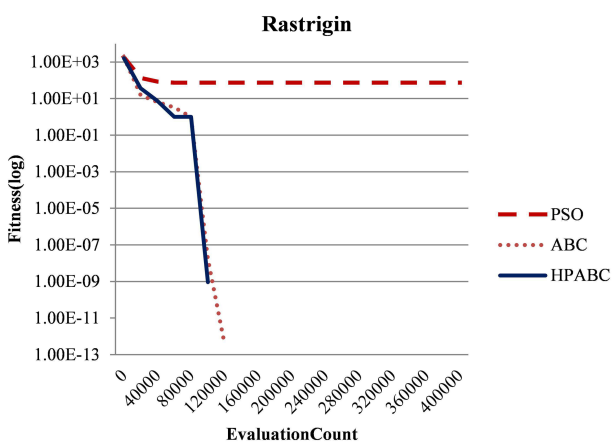

(e)

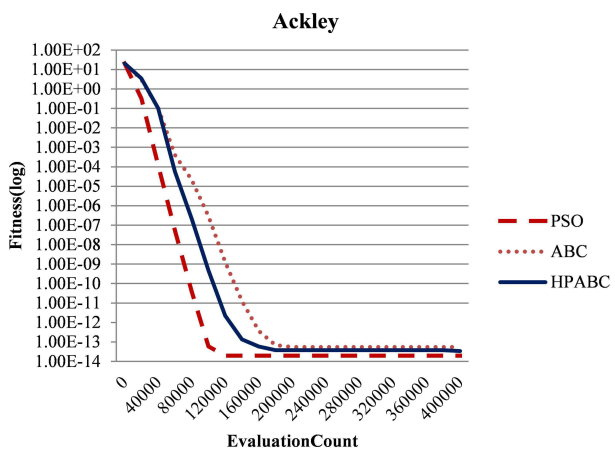

(b)

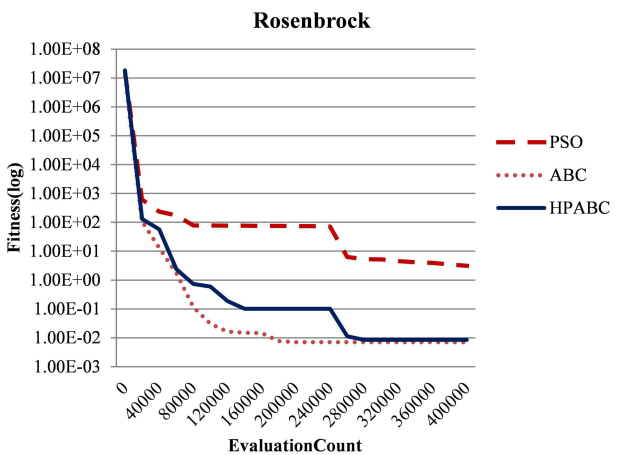

(d)

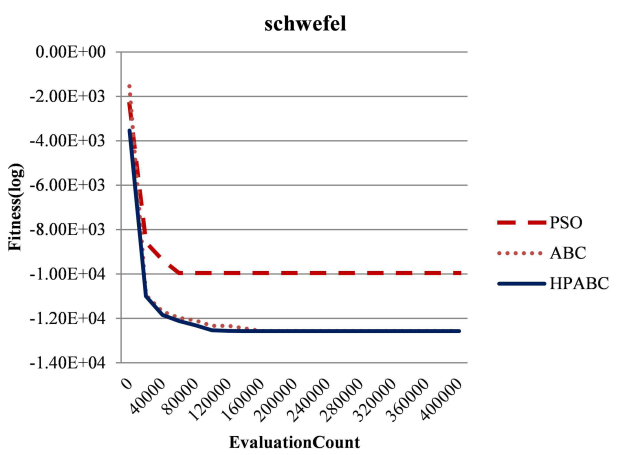

(f)

Fig. 1. (Color online) The convergence performance of the algorithms ABC, PSO and HPABC on numerical functions for 400000 Function Evaluation (FEs) and for dimension $D=30$. (Sphere $\mathrm{FEs}=66000)$. 
is also clear that the HPABC represents a hybridized algorithm. The hybridization features of the HPABC can be counted explicitly by looking at Table 5. Overall, the proposed HPABC algorithm has significantly improved from the original ABC algorithm, especially on the multimodal with separable variables functions. Also the results implicitly illustrate that the HPABC algorithm is a fast algorithm with efficient results where $\mathrm{HPABC}$ outperformed $\mathrm{ABC}$ as well as PSO algorithms in 9 out of 12 tests (see Table 5) in terms of accuracy. This indicates that HPABC is more accurate by $75 \%$. Similarly, HPABC showed better time records in comparison to $\mathrm{ABC}$ algorithm where the total time consumed by $\mathrm{ABC}$ algorithm and HPABC algorithm is $106.2462,31.27269 \mathrm{~s}$, respectively $(106.2 / 31.3 \sim 3.4$ times faster $)$. Nevertheless, its performance clearly represents the hybridization characteristics between the ABC and PSO algorithm, where the HPABC always fall between them or better than one of them in some result cases, as it can clearly seen in Table 5 and Fig. 1.

\section{Conclusion}

This paper presents a Hybridized ABC algorithm called HPABC, where the Particle movement operation of the PSO algorithm is introduced to the ABC in order to improve the search performance of the ABC. To effectively verify the optimization performance of the new proposed HPABC, we compared it with the classical ABC and PSO algorithms on six benchmark functions. These functions have different characteristics to ensure the validity of the proposed algorithm with such type of numerical functions. The results illustrate that the HPABC is performing very significantly in the specified direction that it is supposed to perform. In other words, it is clearly presenting the hybridization objective which is mainly the advantages of the two hybridized algorithms (ABC and PSO). The HPABC algorithm was better than $\mathrm{ABC}$ algorithm in most of the tested functions in terms of fitness values and incredibly faster than the $\mathrm{ABC}$ algorithm in terms of the execution time. On the other hand, the advantage of the PSO algorithm's particle movement process or the solution improvement process had shown its significance into the HPABC search performance. It is also clear that the HPABC had the capability of fast convergence and to efficiently escape trapping at the local minimum on the contrary to the PSO algorithm. Finally, it can be concluded that HPABC algorithm can be efficiently used for solving numerical optimization functions; yet it is still had some difficulties with the multimodal functions with nonseparable variables (Griewank and Ackley). We suggested that more investigation on the HPABC search strategy is needed as a future work through other different optimization problems.

\section{Acknowledgments}

This research experiment has been funded by Ministry of Higher Education (MOHE) under the Fundamental Research Grant Scheme (FRGS) for "Bio-Inspired 
Optimization Method for Feature Selection of Mass Spectrometry Analysis in Biomarker Identification of Ovarian Cancer" [203/PKOMP/6711268] and this research publication has been funded by Universiti Sains Malaysia under the Research University Cluster Grant Scheme (RUC) for "Reconstruction of the Neural Microcircuitry or Reward-Controlled Learning in the Rat Hippocampus" [1001/PKOMP/ 8630022].

\section{References}

1. D. Karaboga and B. Basturk, Appl. Soft Comput. 8, 687 (2008).

2. S. Das, A. Abraham and A. Konar, Swarm intelligence algorithms in bioinformatics, in Computational Intelligence in Bioinformatics (Springer, 2008), pp. 113-147.

3. R. C. Eberhart and J. Kennedy, A new optimizer using particle swarm theory, in Proc. Sixth Int. Symp. Micro Machine and Human Science, Vol. 1, New York (1995), pp. 39-43.

4. M. Dorigo and L. M. Gambardella, IEEE Trans. Evol. Comput. 1, 53 (1997).

5. K. M. Passino, IEEE Control Syst. 22, 52 (2002).

6. X.-L. Li, Z.-J. Shao and J.-X. Qian, Syst. Eng. Theory Practice 22, 32 (2002).

7. D. Karaboga, An idea based on honey bee swarm for numerical optimization. Technical report, Technical report-tr06, Erciyes university, engineering faculty, computer engineering department (2005).

8. D. Karaboga and B. Akay, Artif. Intell. Rev. 31, 61 (2009).

9. B. Akay and D. Karaboga, Inf. Sci. 192, 120 (2012).

10. D. Karaboga and B. Basturk, Artificial bee colony (abc) optimization algorithm for solving constrained optimization problems, in Foundations of Fuzzy Logic and Soft Computing (Springer, 2007), pp. 789-798.

11. D. Karaboga, B. Akay and C. Ozturk, Artificial bee colony (abc) optimization algorithm for training feed-forward neural networks, in Modeling Decisions for Artificial Intelligence (Springer, 2007), pp. 318-329.

12. H. A. A. Bahamish, R. Abdullah and R. A. Salam, Protein tertiary structure prediction using artificial bee colony algorithm, in Third Asia Int. Conf. on Modelling $\mathcal{E}$ Simulation, 2009. AMS'09 (IEEE, 2009), pp. 258-263.

13. D. Karaboga and C. Ozturk, Sci. Res. Essays 5, 1899 (2010).

14. D. Karaboga and B. Gorkemli, A combinatorial artificial bee colony algorithm for traveling salesman problem, in 2011 Int. Symp. on Innovations in Intelligent Systems and Applications (INISTA) (IEEE, 2011), pp. 50-53.

15. M. Y. SyarifahAdilah, R. Abdullah and I. Venkat, ABC algorithm as feature selection for biomarker discovery in mass spectrometry analysis, in 20124 th Conf. on Data Mining and Optimization (DMO) (IEEE, 2012), pp. 67-72.

16. Z. N. Mahmood, M. Mahmuddin and M. N. Mahmood, Int. J. Mod. Phys.: Conf. Ser. 9, 143 (2012).

17. M. Li, H. Duan and D. Shi, Hybrid artificial bee colony and particle swarm optimization approach to protein secondary structure prediction, in 2012 10th World Cong. on Intelligent Control and Automation (WCICA) (IEEE, 2012), pp. 5040-5044.

18. D. Karaboga and B. Akay, Appl. Math. Comput. 214, 108 (2009).

19. X. Kong, S. Liu, Z. Wang and L. Yong, J. Comput. Inf. Syst. 8, 2367 (2012).

20. X. Yan, Y. Zhu and W. Zou, A hybrid artificial bee colony algorithm for numerical function optimization, in 2011 11th Int. Conf. on Hybrid Intelligent Systems (HIS) (IEEE, 2011), pp. 127-132.

21. M. S. Kiran and M. Gündüz, Int. J. Innov. Comput. Inf. Control 8, 6107 (2012). 
22. J. J. Jamian, M. N. Abdullah, H. Mokhlis, M. W. Mustafa and A. H. A. Bakar, J. Appl. Math. (2014).

23. H. Shayeghi and A. Ghasemi, Energy Convers. Manage. 79, 344 (2014).

24. R. Murugan and M. R. Mohan, J. Eng. Appl. Sci. 7, 2012.

25. B. Li, L. Gong and Y. Yao, On the performance of internal feedback artificial bee colony algorithm (if-abc) for protein secondary structure prediction, in 2013 6th Int. Conf. on Advanced Computational Intelligence (ICACI) (IEEE, 2013), pp. 33-38.

26. B. Li, L.-G. Gong and W.-L. Yang, Scientific World J. (2014).

27. Z. N. M. Alqattan and R. Abdullah, Artificial bee colony optimization algorithm with crossover operator for protein structure prediction, in Soft Computing Applications and Intelligent Systems (Springer, 2013), pp. 147-157.

28. X. Zhang, S. L. Ho and W. N. Fu, IEEE Trans. Magnetics 50, 737 (2014).

29. M. S. Kiran and A. Babalik, J. Comput. Commun. 2, 108 (2014).

30. M. A. Abido, Particle swarm optimization for multimachine power system stabilizer design, in Power Engineering Society Summer Meeting, 2001, Vol. 3 (IEEE, 2001), pp. $1346-1351$.

31. M. A. Abido, IEEE Trans. on Energy Conver. 17, 406 (2002).

32. T. Sousa, A. Silva and A. Neves, Parallel Comput. 30, 767 (2004).

33. S. L. Ho, S. Yang, G. Ni, E. W. C. Lo and H.-C. C. Wong, IEEE Trans. Magnetics 41, 1756 (2005).

34. M. Nasri, H. Nezamabadi-Pour and M. Maghfoori, World Acad. Sci. Eng. Technol. 26, $211(2007)$.

35. B. Liu, L. Wang and Y.-H. Jin, IEEE Trans. Syst. Man Cybern. B: Cybern. 37, 18 (2007).

36. A. Chander, A. Chatterjee and P. Siarry, Expert Syst. Appl. 38, 4998 (2011).

37. U. Guvenc, S. Duman, B. Saracoglu and A. Ozturk, Elektronika ir Elektrotechnika 108, 109 (2011).

38. T. Navalertporn and N. V. Afzulpurkar, Swarm Evolut. Comput. 1, 97 (2011).

39. M. Mandal and A. Mukhopadhyay, A multiobjective PSO-based approach for identifying non-redundant gene markers from microarray gene expression data, in 2012 Int. Conf. on Computing, Communication and Applications (ICCCA) (IEEE, 2012), pp. 1-6.

40. M. Mandal and A. Mukhopadhyay, A PSO-based rank aggregation algorithm for ranking genes from microarray data, in Proc. 17th Panhellenic Conf. on Informatics (ACM, 2013), pp. 166-173.

41. S. Talatahari, M. Kheirollahi, C. Farahmandpour and A. H. Gandomi, Neural Comput. Appl. 23, 1297 (2013).

42. J. Yadav and R. Dubey, Int. J. Rev. Electron. Commun. Eng. 1, 48 (2013).

43. F. Zhao, G. Li, C. Yang, A. Abraham and H. Liu, Neurocomputing 132, 68 (2014).

44. M. Mandal and A. Mukhopadhyay, Int. J. Parallel, Emergent Distrib. Syst. (ahead-ofprint):1 (2014).

45. Z. N. M. Alqattan and R. Abdullah, A comparison between artificial bee colony and particle swarm optimization algorithms for protein structure prediction problem, in Neural Information Processing (Springer, 2013), pp. 331-340.

46. G. Zhu and S. Kwong, Appl. Math. Comput. 217, 3166 (2010).

47. Z. N. M. Alqattan and R. Abdullah, Artificial bee colony optimization algorithm with crossover operator for protein structure prediction, in Soft Computing Applications and Intelligent Systems (Springer, 2013), pp. 147-157.

48. N. K. Garg, S. S. Jadon, H. Sharma and D. K. Palwalia, Gbest-artificial bee colony algorithm to solve load flow problem, in Proc. 3rd Int. Conf. on Soft Computing for Problem Solving (Springer, 2014), pp. 529-538. 